Marquette University

e-Publications@Marquette

College of Nursing Faculty Research and

Publications

Nursing, College of

$12-1-2007$

\title{
Dyadic Relationship Scale: A Measure of the Impact of the Provision and Receipt of Family Care
}

Margaret Sebern

Marquette University, margaret.sebern@marquette.edu

Carol J. Whitlatch

Margaret Blenkner Research Institute, Benjamin Rose Institute

Accepted version. The Gerontologist, Vol. 47, No. 6 (December 2007): 741-751. DOI. (C) 2007 Oxford University Press. Used with permission. 


\title{
Dyadic Relationship Scale: A Measure of the Impact of the Provision and Receipt of Family Care
}

Authors: Margaret D. Sebern, $\mathrm{PhD}, \mathrm{RN},{ }^{1}$ and Carol J. Whitlatch, $\mathrm{PhD}^{2}$

\begin{abstract}
Purpose: This study evaluated the psychometric properties of the Dyadic Relationship Scale (DRS), which measures negative and positive dyadic interactions from the perspective of both the patient and the family caregiver. An important aspect of evaluating the DRS was that it be statistically sound and meaningful for both members of the dyad. Design
\end{abstract} and Methods: The study used a cross-sectional design. Survey packages were mailed to home health care patients and their family caregivers. The unit of analysis was the dyad, and exploratory and confirmatory factor analyses were conducted. We examined the reliability, discriminant, and concurrent validities of the instrument. Results: The data supported a twofactor DRS that included negative dyadic strain (patient ( $\alpha=.84$; caregiver $(\alpha=.89)$ and positive dyadic interaction (patient $\alpha=.86$; caregiver $(\alpha=.85$ ). The analysis supported the DRS's construct, discriminant, and concurrent validity, as well as its reliability for both patients and family caregivers. Implications: Using the DRS to measure the impact of family care on positive and negative interactions inclusive of patients and caregivers can assist in identifying areas of difficulty and guide interventions to improve outcomes for both members of the dyad.

An increasing body of research has documented that when a relative requires care due to illness or disability, the preference of most adults is for that care to be provided by family or friends (Cutrona \& Cole, 2000). This preference is consistent for individuals who require care and for those who provide the care (Kellett, 1999a). Family care can occur across the life span and refers to unpaid assistance to a patient by family and friends in the patient's place of residence (Archbold et al., 2001). A family caregiver can be any family member or friend (broadly defined) who provides assistance to a patient. Family care involves complex interactions that can be stressful and lead to poor physical and mental health outcomes for both providers and receivers of care (Beach et al., 2005). Positive aspects of family care also exist, such as improved self-worth, sense of meaning, and adaptation to chronic illness (Kramer, 1997; Reinhardt, 2001). It is important for clinicians and researchers to understand the positive and negative aspects of family care because of the impact of these factors on outcomes for both caregivers and patients. Moreover, the care provided by family members assists patients to live in communities of their preference.

1 Sebern \& Witlatch 
The concept of family care intrinsically involves two individuals in a close relationship, yet the majority of family care research and intervention studies have focused on one person from the care dyad (Martire, Lustig, Schulz, Miller, \& Helgeson, 2004). In the medical and nursing literature, the focus is more patient centered, and the providers of family care are often overlooked by researchers studying patient outcomes (Lauver et al., 2002; National Institute of Nursing Research, 2004). Within the gerontology and caregiving literature, the focus is on caregiver outcomes, with less attention paid to patient outcomes (Martire et al., 2004). By focusing only on one or the other member of the care dyad, investigators neglect important information about the reciprocal influence of one person on the other. Researchers have suggested that the study of family care could be advanced by including both members of the dyad, developing constructs that capture the positive and negative aspects of family care, and developing psychometrically sound tools that measure these constructs (Kenny, Kashy, \& Cook, 2006; Kramer, 1997; Reis \& Collins, 2000; Whitlatch, Feinberg, \& Tucke, 2005).

The present study addressed two themes related to the provision of family care for adults with chronic conditions. First, we included both members of the family care dyad. Second, we focused on evaluating the psychometric properties of the Dyadic Relationship Scale (DRS), which measures positive and negative aspects of the dyadic relationship intrinsic to family care. Experts developed and revised this tool to measure appraisals of family care relationships from the perspective of each individual (Bass, Tausig, \& Noelker, 1989). In addition to examining the psychometrics of the DRS, we examined the association between the DRS and depressive symptoms.

Family care as a dyadic process is based on the premise that each participant affects and is affected by the other (Gayle \& Preiss, 2002; Kenny et al., 2006). In addition to dyadic factors, another important influence on family care is a family's history and patterns of interdependence. Families have experiences providing assistance to members and develop interpersonal patterns that are not easily changed (Gaugler et al., 2002). For example, family members may see themselves as primarily parents, spouses, friends, or other relations. For some, these relationships lead naturally to caring for one another because of the nature of belonging to a family with mutual concern for its members. However, for others that may not be true. Past relationships, either positive or negative, will influence willingness to provide assistance in the present. Families with a willingness to provide assistance to members in need may nevertheless lack the knowledge, skill, and/or ability to provide the care.

One theoretical framework for studying family care is social support, specifically the influence of interpersonal relationships on health status (Reis \& Collins, 2000; Wills, 1985). 
Using this framework, Bass and colleagues (1989) found that supportive family or friends have the capacity to alter the relationship between need for assistance in chronic illness and relationship strain. Supportive relationships may alter the negative effects of physical impairment on strain by reducing the consequences of a patient's impairment (Bass et al., 1989). These authors defined relationship strain as deterioration in the quality of a patient/family member relationship as a result of caregiving. Bass and colleagues assumed that the experience of providing and receiving care is a function of perceptions and appraisals of the situation rather than an outside objective viewpoint. These researchers adapted and revised a previous version of the DRS (Poulshock \& Deimling, 1984) that originally drew items and constructs from earlier measures of caregiver burden and stress (Zarit, Reever, \& BachPeterson, 1980).

Reis and Collins (2000) embedded social support in an interpersonal context and suggested that three types of phenomena are critical to understanding social support as an interpersonal process: (a) abstract attributes, like synthesis of past, present, future, expectations, identities, and patterns of communication; (b) interpersonal predispositions that influence interactions and relationships, such as interdependence orientation in communal relationships; and (c) concrete supportive interactions that take place. These authors argued that a change in one of these phenomena will influence a change in the other components. They suggested that long-term relationships provide multiple opportunities for reversing the role of support seeker and provider, and support is more likely to the extent that a relationship is interdependent, responsive, and affectively positive. Reis and Collins also suggested that positive and negative affect represent independent qualities in a relationship.

Reinhardt (2001) examined the effect of positive and negative aspects of family care, both received and provided, on adaptation to chronic physical impairment in 570 patients with age-related vision loss. Findings indicated that patients who received affective support had less depressive symptoms, greater life satisfaction, and better adaptation. Visually impaired patients who provided greater affective support to their family members also had higher life satisfaction. Both the receipt and the provision of negative exchanges with family members were associated with greater depressive symptoms.

Additional research has suggested that recipients of care provide substantial assistance to their caregivers, and these acts of reciprocity are important to well-being (Davey \& Eggebeen, 1998; Liang, Krause, \& Bennett, 2001). Liang and colleagues suggested that negative aspects of received support are helping distress (feeling mental and emotional strain when helped) and negative interaction (feeling others are being critical of you and prying into personal affairs). For 
care recipients, low relationship quality and not being able to reciprocate when receiving assistance predicted depression (Davey \& Eggebeen, 1998). In contrast, opportunities to reciprocate within a family contributed to positive aspects. Research has also reported positive aspects of family care for caregivers. For example, spouses who increased the amount of assistance provided to a disabled partner had lower anxiety and depression (Beach, Schulz, Yee, \& Jackson, 2000). Moreover, research has indicated that family involvement in care can provide caregivers with a sense of meaning, purpose, and control (Kellett, 1999b; Lawton, Kleban, Moss, Rovine, \& Glicksman, 1989).

The findings from these studies highlight the importance of measuring (a) positive and negative aspects of family care and (b) the influence of these aspects on the well-being of both members of the care dyad. By understanding and measuring the positive and negative aspects of family care, clinicians and researchers could reconceptualize how they assess members of the dyad, work more effectively with the dyad, and guide the testing of interventions to improve outcomes for both members of the dyad. The focus of this study was the evaluation of the psychometric properties of a revised version of the original DRS using a sample of home health family care dyads. An important aspect of the evaluation was to validate that the DRS was statistically sound and meaningful for both the caregivers and care recipients. Specifically, we examined (a) the scale's reliability (internal consistency), (b) the construct validity of the caregiver and patient versions of the DRS, and (c) discriminant and concurrent validities. Based on propositions by Kramer (1997) and Reis and Collins (2000) that positive and negative affect represent independent phenomena, we expected to find a positive correlation between negative dyadic strain and depressive symptoms for both members of the dyad, and no correlation between positive interaction and depressive symptoms.

\section{Methods}

\section{Sample}

A Medicare-certified home health care agency serving both urban and rural populations in a midwestern state provided access to patients and caregivers for this study. Personnel from the agency's information system department selected patients from their databases who met study criteria. Criteria for inclusion were being older than 18 years of age, having intact cognitive ability and vision, and living with a person who provided unpaid assistance. Home health care agency staff used items from the Outcome and Assessment Information Set (OASIS) to determine if the patient was eligible to participate in the study (Centers for Medicare \& Medicaid Services, 2007). Medicare-certified home health care agencies are mandated to use OASIS for 
outcome assessments. Cognitive status was assessed with OASIS Item M0 560-Cognitive Function. Patients were eligible if they had a rating of 0 (alert/oriented, able to focus and shift attention, comprehends and recalls task directions independently) or 1 (requires prompting only under stressful or unfamiliar conditions). Vision was assessed with OASIS Item M0 390-Vision With Corrective Lenses if the Patient Usually Wears Them. Patients were eligible if they had a rating of 0 (sees adequately in most situations; can see medication labels, newsprint). The criteria that the patient lived with someone who provided them with assistance was measured with OASIS Item M0 350-Assisting Person(s) Other Than Home Care Agency Staff. Patients were eligible if a person assisting them was residing in the home. Agency personnel identified 1,107 eligible cases. From the list of eligible patients, 579 cases were randomly selected using a table of random numbers and invited to participate in the study.

\section{Procedures}

We obtained institutional review board approval from university and health care organizations. The study used a cross-sectional design. Survey packages were mailed to home health care patients who were identified as having an informal caregiver. The identities of caregivers were unknown to the researcher; therefore, the patient participants were instructed to give the caregiver survey to whomever assisted them the most. Each survey package included a patient and a caregiver version of the DRS, the Center for Epidemiologic Studies-Depression scale (CES-D), demographic questions, and a measure of functional status; written instructions; and a self-addressed, stamped return envelope.

Both members were encouraged to participate. To support participation, we mailed a prenotification letter prior to mailing the survey package, clipped a $\$ 2$ token of appreciation to each survey, and sent a post card reminder 1 week after the survey was mailed. At 4 weeks we sent a second survey to non-responders, and at 6 weeks non-responders received a follow-up phone call inquiring about the survey. In a few cases participants provided a reason for not participating; common reasons were language differences (e.g., patient spoke Russian or Spanish), patient hospitalized, too busy, and wrong contact information. About $4 \%$ of patients ( $n$ $=18$ ) did not participate due to death prior to receiving the survey.

\section{Instruments}

The DRS has been revised since it was first presented by Poulshock and Deimling (1984). The original 11-item scale, which drew upon previous measures of caregiver stress and burden (Zarit et al., 1980), assessed the caregiver's perspective of dyadic and family relationship strain associated with providing care to older family members. More recently, experts have added items that assess the positive aspects of providing care and the patient's

\section{Sebern \& Witlatch}


perception of the dyad's relationship, and deleted items that reflect the broader family relationships (Bass, Clark, Looman, McCarthy, \& Eckert, 2003; Clark, Whitlatch, \& Tucke, 2005; Feinberg, Whitlatch, \& Tucke, 2000; Menne, 2006). DRS items assess a variety of potential relationship stressors: "Because of my health condition, I felt angry toward her/him" (patient version) and "Because of helping my family member, I felt angry toward her/him" (caregiver version). An example of an item measuring patient relationship quality is "Because of my health condition, I felt closer to her/him than I have in awhile." For this study we administered both patient and caregiver versions that included a four-option response $(1=$ strongly disagree, 2 = disagree, 3 = agree, 4 = strongly agree). The patient version (10 items) and the caregiver version (11 items) have two subscales: dyadic strain and positive dyadic interaction. Higher scores on each of these scales indicate higher levels of strain and positive interaction, respectively.

We measured depressive symptoms using the CES-D, a 20-item, four-option response scale (Radloff \& Teri, 1986). In the current study, Cronbach's alphas for the CES-D were .89 (patients) and .88 (caregivers). The validity and reliability of the CES-D is well established, and researchers have used the measure widely in health- and population-based research (Eaton, VandeCreek, \& Jackson, 2001). Higher scores on the CES-D indicate higher levels of depressive symptoms, and a cutoff score of 16 or higher is commonly used to indicate the presence of depressive symptoms (McDowell, 2006).

We defined patient functional status as the patient's and caregiver's subjective perceptions of the patient's limitation in normal activity. We measured patient functional status with the Karnofsky Index of Performance Status (KPS) (McDowell, 2006). The KPS has 10 categories describing a patient's limitation in normal activity (e.g., patient's ability to work, performance of normal activities, and need for assistance). KPS scores range from 1 (s/he is able to carry on with normal activity including work) to 10 (his/her condition is a fatal process progressing rapidly). Higher KPS scores indicate the patient requires more assistance from caregivers.

\section{Approach to Psychometric Analysis of Dyadic Data}

We used methods of dyadic analysis proposed by Kenny and colleagues (Campbell \& Kashy, 2002; Gonzalez \& Griffin, 1999; Kenny et al., 2006). The dyad was the unit of analysis: each variable was contained twice in the data set (i.e., once for each dyad member). Based on the structure identified in the exploratory factor analysis (EFA), we conducted a confirmatory factor analysis (CFA) to confirm the factor structure. Even though dyadic data are dependent (i.e., indicators within the dyad share variance), it is possible with CFA and structural equation 
modeling to correlate the errors of the indicators to model dependency in dyadic data (Kenny et al., 2006). We allowed the errors of the indicators to correlate in the CFA; thus, the parameters modeled the dependency in the dyad data.

Once the DRS factor structure was supported, we conducted a CFA with background demographic variables to determine whether population heterogeneity contributed to measurement invariance. We used multiple indicator multiple cause (MIMIC) models to evaluate background variables. MIMIC models evaluated the separate associations between age, gender, kin relationship, length of relationship, and caregiver or care recipient role on the DRS scales. The results of significant covariates on the DRS scales would indicate differential item functioning. We used a structural equation model to evaluate CES-D as a function of the DRS dimensions. An association between CES-D and dyadic strain would support the concurrent validity of the DRS.

For EFA, CFA, and CFA with background variables (MIMIC) we used the MPlus software package (Muthén \& Muthén, 2005). MPlus was specifically designed to work with categorical data. MPlus handles dependent dyadic data consistent with David Kenny's approach by correlating the errors of the indicators (Maydeu-Olivares, 2000; Muthén \& Muthén, 2000). With the MIMIC models we regressed the DRS factors on the background variables age, gender, kin relationship, length of relationship, and caregiver or care receiver role. We evaluated the association between DRS scales and patient and caregiver depressive symptoms with structural equation modeling.

Finally, we estimated the reliability and discriminant validities of the dyadic relationship scales. We estimated scale reliability using the ratio of the sum of the item loadings squared times the variance of the scale over the sum of the item loadings squared times the variance of the scale plus the sum of the item variances. We estimated the reliability using the following equation:

$$
\rho_{\eta}=\frac{\left[\sum_{i=1}^{p} \lambda_{y i}\right]^{2} \operatorname{var}(\eta)}{\left[\sum_{i=1}^{p} \lambda_{y i}\right]^{2} \operatorname{var}(\eta)+\sum_{i=1}^{p} \operatorname{var}\left(\varepsilon_{y i}\right)}
$$

where $\eta=$ the specific scale, $\lambda=$ the factor loading, and $\varepsilon=$ the residual term (Dillon \& Goldstein, 1984).

To estimate the DRS's discriminant validity we used Fornell and Larker's (1981) suggestion that convergent latent variables (LVs) should have measures that contain more than 
$50 \%$ explained or common variance in the factor analytic sense. These authors proposed using the average variance extracted (AVE) statistic as a measure of convergent validity. AVE is the amount of variance captured by the LV in relation to the amount of variance due to its measurement error (Dillon \& Goldstein, 1984). In different terms, AVE is a measure of the errorfree variance of a set of items. One can also use AVE to gauge discriminant validity (Fornell \& Larker, 1981). If the squared correlation between two LVs is less than either of their individual AVEs, this suggests the LVs each have more internal (extracted) variance than variance shared between the LVs. If this is true for the target LV and all of the other LVs, this suggests the discriminant validity of the target LV.

\section{Missing Data}

Most missing data were due to skipped pages or randomly omitted answers, and we assumed these to be missing at random. We deleted cases if more than $15 \%$ (i.e., two) of DRS items were missing. We deleted 33 patient cases and 18 caregiver cases due to missing more than $15 \%$ of data. We used multiple imputation methods to impute missing data by imposing a probability model on the complete data and observed and missing values (Schafer, 1997). The patient response rate was 39\% ( $n=227)$, and the caregiver response rate was 39\% $(n=228)$. We used only matched pairs of patients and caregivers for this analysis. The sample size for matched dyads was 200 (35\% response rate), and we used these data to examine the factor structure, construct validity, and reliability of the DRS.

There was no significant difference in participation based on patient illness as measured by International Classification of Disease- 9 codes, $\chi^{2}(12, n=542)=14.97, p=.24$. However, there was a significant difference in the agreement-to-participate rate based on the home health care office serving the patient. Offices serving more urban and populated areas had participation rates of $16 \%$ to $20 \%$, and suburban and rural office participation rates were $40 \%$ to $50 \%, \chi^{2}(10)=22.0, p=.015$.

We compared the patients who participated without a partner to the patients with a partner on the variables age, gender, length of relationship, KPS, CES-D, strain, and positive interactions. These comparisons of patients with and without a partner participating were not significant, except for the strain variable. Patients without a partner participating in the survey reported significantly more dyadic strain than patients participating with a partner ( $T=2.7,95 \%$ confidence interval $[\mathrm{Cl}]=0.3-2.3, p<.006)$. We also used the same variables to compare the group of family caregivers who participated without a patient and caregivers who participated with a patient. The only significant difference for the caregiver groups was on the KPS variable. 
Caregivers who participated alone reported that the patient required more assistance than did the group of caregivers who participated with the patient $(T=3.0,95 \% \mathrm{Cl}=0.47-2.1, p<.002)$.

\section{Results}

Patients ranged in age from 21 to 95 years $(M=66$ years, $S D=14$ years; see Table 1$)$. Only $3.5 \%$ of patients were between 21 and 40 years old. There was no significant relationship between age and the DRS dimension (see Table 2). Because of the lack of a significant relationship between age the DRS scales, and due to the observation that family care occurs across the life span, we believed it was important to include chronically ill younger patients in our results. A total of $42 \%$ of the patients were female. A large percentage (94\%) of patients were White, and less than $1 \%$ were African American.

The most frequent patient medical conditions were circulatory $(27 \%)$, musculoskeletal (13\%), neoplasm (9\%), and diseases of the skin (8\%). A moderate number of patients had completed college or graduate degrees (19\%); however, more had only completed high school (61\%). The median KPS score was 4 (range 1-10) based on patient self-report and 5 (range 110) based on caregiver self-report, indicating that more than half of the patient participants required occasional assistance. The KPS scores were not significantly different between patients and caregivers (Wilcoxon $Z=-0.78, p=.43$ ). The intraclass correlation between the patient and caregiver KPS ratings was $0.75(95 \% \mathrm{Cl}=0.68$ to 0.80$)$, suggesting that the patient and caregiver perceived the patient's need for assistance similarly.

Patients must meet Centers for Medicare \& Medicaid Services criteria for being homebound (unable to leave the home without considerable assistance) to receive home care service. When patients do not meet Medicare criteria for homebound or are medically stable, they are discharged from home care services to the care of their families. When participants were asked if they were currently receiving home care services, $76 \%$ ( $n=180)$ of patients said they were not currently receiving home care services. Thus, many of the participants were discharged from home care services when they completed the surveys. Their no longer meeting Medicare criteria for homebound status may have been reflected in the lower KPS scores.

The age of caregivers ranged from 18 to 93 years $(M=65$ years, $S D=14$ years). A total of $61 \%$ of caregivers assisting patients were female. The majority of caregivers were White (91\%), whereas $2 \%$ were African American and less than $2 \%$ were American Indian. Hispanic ethnicity was reported by $1 \%$ of patients and caregivers. In all, $20 \%$ of family participants had completed college or graduate degrees, and more had completed only high school (59\%). 
Most patients ( $81 \%$ ) were related to their caregiver as a spouse or partner, $7 \%$ were parents, $7 \%$ were adult children or friends of the caregiver, and $4 \%$ were related as other. Caregivers were related in a corresponding manner to the patients: $81 \%$ spouses/partners, $9 \%$ children or friends, $4 \%$ parents, and $4 \%$ other. Members of the dyads had known each other on average for 41 years. In all, $42 \%$ of the patients $(n=85)$ and $28 \%$ of caregivers $(n=57)$ reported that they were both a giver and a recipient of care.

\section{Exploratory Analysis of the DRS Factor Structure}

We evaluated nonindependence of the data with interclass correlations. Interclass correlations and Cls are reported in Table 2. Most of the interclass correlations were low to moderate (range $0.13-0.48$ ). DRS Item 6 (i.e., "I have had more patience than I had in the past") did not appear to be a dependent item within the dyads.

We used EFA to estimate the factor structure and then used CFA to confirm the hypothesized structure. For the EFA, we combined the patient and caregiver data to model the dependency between the two members of the dyad and analyzed one-, two-, and three-factor solutions using the following criteria: (a) eigenvalue greater than 1, (b) items loaded on the same factor for patients and caregivers $(>0.40)$, (c) items did not load on two or more factors $(>0.30)$, and (d) interpretability of factors.

A two-factor solution represented the patient and caregiver data adequately in that all items loaded on the same factors with factor loadings from 0.41 to 0.85 , and the factors were interpretable. The six positive items loaded on the first factor for both patients and caregivers (positive dyadic interaction). The positive items indicated a person felt closer, had improved communication, had more patience, and learned good things about oneself as a result of family care. The negative patient and caregiver items loaded on the second factor (dyadic strain). The dyadic strain items indicated feelings of anger, strain, and resentment resulting from family care.

\section{Confirming the Factor Structure}

CFA supported the hypothesized factor structure. In the CFA we defined the correlated variables within dyads and specified the model for (a) the patient positive dyadic interaction and dyadic strain and (b) the caregiver positive dyadic interaction and dyadic strain. MPlus modeled the dependency across the dyad and incorporated this into the modeling. The patient factor loadings ranged from 0.49 to 0.88 for positive dyadic interaction and from 0.58 to 0.86 for dyadic strain (see Table 3). One item on the caregiver strain subscale is not included on the patient strain scale (i.e., "The patient makes more demands than needed"). The caregiver factor loadings ranged from 0.41 to 0.85 for positive dyadic interaction and from 0.66 to 0.91 for dyadic strain (see Table 3).

10 Sebern \& Witlatch 
The variance explained for each factor was as follows: patient dyadic strain, $29 \%$; caregiver dyadic strain, 24\%; patient positive dyadic interaction, 44\%; and caregiver positive dyadic interaction, $28 \%$. The fit indices supported the fit between the data and the model. For the CFA model, the root mean square error of approximation (RMSEA) was 0.07 and the comparative fit index (CFI) was 0.96 . Kenny and colleagues (2006) suggested that a RMSEA 0.10 or less indicates a reasonable error of approximation and a CFI above 0.95 indicates an adequate fit. The chi-square/degrees of freedom ratio was 2/1. Kline (2005) recommended that judgments of model fit be based on a chi-square/degrees of freedom ratio of <3.0.

The patient DRS dimensions had small to moderate correlations with the caregiver DRS dimensions. For example, patient dyadic strain was moderately correlated with caregiver dyadic strain $(r=.46, p<.05)$. The patient positive dyadic interaction factor was moderately correlated with the caregiver positive dyadic interaction factor $(r=.48, p<.05)$. Patient positive dyadic interaction was moderately correlated with the patient dyadic strain $(r=-.40, p<.05)$ but not with caregiver dyadic strain $(r=-.14, n s)$. In a similar pattern, caregiver positive dyadic interaction was moderately correlated with the caregiver dyadic strain $(r=-.32, p<.05)$. These correlations suggested that the person's own positive and negative dimensions were correlated, and the within-dyad positive and negative dimensions were related to the respective positive and negative dimensions of the other person.

We used MIMIC models to evaluate the separate associations between the background variables age, gender, kin relationship, length of relationship, and caregiver and care recipient role and the DRS scales. With the MIMIC models we regressed the DRS factors on the background variables. None of these background variables had a significant relationship with the DRS dimensions. These findings supported the DRS's measurement invariance across age, gender, kin relationship, length of relationship, and the role of caregiver and recipient.

\section{Concurrent Validity of the DRS With the CES-D}

In the final aspect of examining the construct validity, we used a structural equation model to evaluate the concurrent validity of the DRS scales by regressing the CES-D on the DRS dimensions. Concurrent validity was supported for dyadic strain. The partial standardized regression coefficient between patient CES-D with patient dyadic strain was $0.53(p<.05)$. For the caregiver, the partial standardized regression coefficient between caregiver CES-D and dyadic strain was $0.47(p<.05)$.

Unexpectedly, the partial standardized regression coefficient for positive interaction with patient CES-D was positive and significant at $0.20(p<.05)$, indicating that positive interactions had a positive association with depressive symptoms (see Table 4). The partial standardized 
regression coefficient between caregiver CES-D and caregiver positive dyadic interaction was not significant at 0.05 ( $p>.05$; see Table 5). Patients' mean CES-D score was 16.3 (SD =11.8, $95 \% \mathrm{Cl}=14.6-18.0)$, and caregivers' mean CES-D score was $11.3(S D=9.1,95 \% \mathrm{Cl}=10.0$ 12.6). There was a significant difference between patient and caregiver CES-D scores (mean difference $=4.9,95 \% \mathrm{Cl}=2.8-7.0, t=4.6, p=.000$ ), indicating that patients reported higher levels of depressive symptoms compared to their caregivers.

The fit statistics supported the structural equation model for CES-D and DRS data. The RMSEA was 0.07 , and the CFI was 0.96 . The DRS dimensions explained $23 \%$ of the variance in patient depressive symptoms and $21 \%$ of the variance in caregiver depressive symptoms. These findings partially supported the concurrent validity of the DRS, in that dyadic strain was positively associated with depressive symptoms for both patients and caregivers. However, there was a differential influence of positive dyadic intervention: For the patient, positive interaction was associated with depressive symptoms, and this was not supported for the caregiver.

Using the AVE statistic (Fornell \& Larker, 1981), our results indicated that the two DRS dimensions had convergent and discriminant validity for both groups. The AVE for the two patient dimensions ranged from .51 to .58 , and the AVE for the two caregiver dimensions was .58 to .63. The two DRS dimensions also demonstrated discriminant validity for both groups: Patient squared correlation between strain and positive interactions was .16 (less than the AVE of .58 for strain or .51 for positive interactions). Caregiver squared correlation between strain and positive interactions was .104 (less than the AVE of .63 for strain or .50 for positive interactions.

\section{Reliability of Subscales}

The item means ranged from 1.9 (caregiver dyadic strain) to 2.9 (patient and caregiver positive dyadic interaction). Estimates of internal consistency reliability in both groups for the two subscales scores were adequate. Internal consistency for the scales was .86 (patient positive dyadic interaction), .84 (patient dyadic strain), .85 (caregiver positive dyadic interaction), and .89 (caregiver dyadic strain).

\section{Discussion}

We collected data from both members of the dyad, and the unit of analysis was the dyad. We modeled nonindependence of data consistent with Kenny and colleagues' approach for analysis of dyad data (Campbell \& Kashy, 2002; Kenny et al., 2006). These researchers stressed the importance of considering the interdependence that exists between dyad members. 
Score interdependence is a critical issue due to mutual influences within dyads. Mutual dyad influences suggested by Kenny and colleagues are (a) compositional effects, in that similar people are attracted to each other; (b) partner effects, in that the characteristics of one person affect the outcomes of the other; (c) mutual influence, in that outcomes for each member of the dyad directly affect the other through a feedback process; and (d) common fate, or the extent that both are exposed to the same causal factor. The interclass correlation between dyads on the DRS items suggests that the dyads had heightened similarity in how they experienced the effects of providing and receiving assistance. We modeled this interdependence of the dyad data by allowing the variance terms to correlate in our models. The parameters resulting from our analysis controlled for shared variance.

The present research provides statistical support through CFA that the DRS consists of two factors-dyadic strain and positive dyadic interaction-that providers and receivers of care attribute to family care. The factor structure of the DRS is invariant across dyads for age, gender, kin relationship, length of relationship, and caregiver or care recipient role. The discriminant validity test for the DRS argues for viewing the impact of providing and receiving care as two dimensions. Fit statistics support the adequacy of the fit between the data and models. Reliability was acceptable for both dyadic strain (patient $\alpha=.84$; caregiver $\alpha=.89$ ) and positive dyadic interaction (patient $\alpha=.86$; caregiver $\alpha=.85$ ).

The DRS explained $23 \%$ of the variance in patient depressive symptoms and $21 \%$ of the variance in caregiver depressive symptoms. Dyadic strain was significantly associated with depressive symptoms for both members of the dyad. These findings are consistent with previous research suggesting that dyadic strain mediates the negative or stressful effects of caregiving on the health status of both members of the dyad (Bass et al., 1989; Beach et al., 2000; Martire et al., 2004). However, there can be a reciprocal relationship between strain and depressive symptoms, in that depressive symptoms can impact relationship strain. Longitudinal studies are needed to establish any causation between dyadic strain or positive interaction and depressive symptoms. The patients in the current study had a chronic illness, the most prevalent being cardiovascular. Lebowitz and colleagues (1997) suggested that chronic illness is related to depressive symptoms. Researchers have also reported high rates of depressive symptoms for individuals with heart disease (Sullivan, Levy, Russo, \& Spertus, 2004; Turvey, Schultz, Arndt, Wallace, \& Herzog, 2005).

An unexpected finding was a weak association between patients' positive interaction and depressive symptoms. This finding suggests that even with supportive caregivers, other variables such as chronic illness may contribute to a patient's depressive symptoms. Lebowitz 
and colleagues (1997) suggested that chronic illness is related to depressive symptoms. Researchers have also reported high rates of depressive symptoms for individuals with heart disease (Sullivan et al., 2004; Turvey et al., 2005). It is interesting to note that the average score for patients on the CES-D (16.3) was higher than the average score for noninstitutionalized adults (8.7; McDowell, 2006).

Positive interactions may also be associated with depressive symptoms if patients perceive that they receive more assistance than they provide to the caregiver. Perceptions of overbenefiting in social relationships may contribute to depressive symptoms. Davey and Eggebeen (1998) and Liang and colleagues (2001) found that overbenefiting in a social relationship predicted elders' depressive symptoms, and underbenefiting and more anticipated support were associated with less depressive symptoms.

Bass and colleagues (1989) and Bass, McKee, Deimling, and Mukherjec (1994) suggested that cognitively intact patients are better able to exchange gratitude and appreciation in return for assistance from a family member, and these exchanges may make the relationship more mutually satisfying and less stressful. Helping chronically ill patients learn strategies for reciprocating when they receive assistance may be important to patients' mental health. Taken together, these findings suggest that patients and caregivers experience family care in unique ways and point to the importance of tailoring interventions to their unique needs and measuring outcomes for both.

Several other researchers have posed that there are positive and negative dimensions in family care. Kramer (1997) proposed a two-factor gain-and-strain theory for caregiving, suggesting that there are different correlates to the gain-and-strain experience. For example, strain is likely associated with depressive symptoms, and positive interactions influence satisfaction with the relationship or gain. Krause (2005) found in a longitudinal study with 521 individuals with a self-reported heart problem that negative interactions had a larger impact on health than positive interactions. The types of negative interactions studied included criticism, rejection, violation of privacy, lack of reciprocity, and feelings of anger about an unpleasant social situation. Krause suggested that interpersonal skills such as perspective taking, negotiating opposing views, and finding meaning might mediate the influence of negative interactions on health. Interventions teaching interpersonal skills and ways to find meaning in adversity might reduce dyadic strain and improve outcomes for both patients and family caregivers.

A limitation of the study is that we could not ensure that patients and caregivers completed their surveys independently. Other study limitations were the homogeneous sample, 
the fact that $80 \%$ of dyads were spouses, and the low response rates from central-city home health care patients. Factors that may have contributed to low response rates were problems with accurate addresses, the transient nature of central-city residents who may not have owned their own homes, and the lack of trust related to participating in mailed surveys. Future research with central-city home health care patients might consider having a trusted person, such as a patient's home care nurse, deliver the survey and using telephone or in-person methods of data collection. Generalizability is limited due to the sample characteristics of the primarily Caucasian, cognitively intact adults who had a caregiver residing in the home and who were home health care recipients. However, investigators have reported the same two-factor solution previously in a sample of cognitively impaired older adults (Clark et al., 2005; Menne, 2006).

Patients are discharged from home care when either they are medically stable or they do not meet the Medicare criteria for homebound status (i.e., patient requires considerable human assistance to leave the home). Although $79 \%$ of participants were not currently receiving home care services, this does not mean that there was an end to the caregiving/receiving relationship. After home care discharge, patients continue to manage chronic conditions with the assistance of family and friends. Patients in this study were on average 66 years old, had a chronic illness, lived with someone who provided them with assistance, and reported more depressive symptoms than their caregivers and the general population. When a patient is discharged to the care of family members, both members of the dyad may be at risk for negative health outcomes. Anticipatory planning such as relationship skills training may improve outcomes for both.

Implications for future research are that positive and negative aspects of the dyadic relationship are important to assess from perspectives of both the giver and recipient of care. Interventions targeting positive aspects of the relationship and decreasing strain may be important to improving outcomes. Coaching the patient to reciprocate when assistance is provided may be especially important for the patient's mental health.

Experts could fill a gap in the family care research by targeting interventions to both members of the patient-caregiver dyad (Burg et al., 2005; Whitlatch, Judge, Zarit, \& Femia, in press). For example, in the case of a family dyad managing chronic illness, experts could develop and test interventions to coach both the patient and the caregiver about interpersonal communication skills in the context of chronic illness self-management, decision making, and providing and receiving assistance. The interpersonal communication skills components could include (a) listening and perspective taking, (b) interpreting and responding to symptoms, (c) asking for help, (d) responding to unwanted assistance, (e) expressing emotions and appreciation, and (f) making certain each person understands what the other has said. Margaret 
D. Sebern is currently developing this approach, which offers significant advantages over the traditional approach of coaching the patient without involving the caregiver.

The DRS is a useful tool that can be used in research and practice to evaluate (a) the patient's and caregiver's individual perceptions of the impact of family care on the dyadic relationship and (b) the influence of the dyadic relationship on their physical and mental health outcomes. Reliability and construct validity findings from this study support continued use of the DRS. Several researchers have identified a need for studying family care from the perspectives of both members of the patient-caregiver dyad, with particular attention to how family care affects outcomes for both parties (Martire et al., 2004). Using the DRS offers researchers and practitioners a way to study care interactions inclusive of patients and caregivers, assist in identifying areas of difficulty, and guide interventions to improve outcomes for both members of the dyad.

\section{Notes}

- Funded by National Institute of Nursing Research Grant 5T32 NR07102-03.

- Address correspondence to Margaret D. Sebern, College of Nursing, Marquette University, Clark Hall, Room 351, P.O. Box 1881, Milwaukee, WI 53201. E-mail: marge@sebern.com

- $\quad{ }^{1}$ College of Nursing, Marquette University, Milwaukee, WI.

- ${ }^{2}$ Margaret Blenkner Research Institute, Benjamin Rose Institute, Cleveland, $\mathrm{OH}$.

\section{References}

Archbold, P. G., Baker, J. C., Buckwalter, K. C., Burgio, L., Clark, P. C., Gitlin, L., et al. (20()1). Research in informal caregiving: State of science workgroup meeting summary. Washington, DC: National Institute of Nursing Research.

Bass, D. M., Clark, P. A., Looman, W. J., McCarthy, C.A., \& Eckert, S. (2003). The Cleveland Alzheimer's managed care demonstration: Outcomes after 12 months of implementation. The Gerontologist, 43, 73-85.

Bass, D. M., McKee, J., Deimling, G. T., \& Mukherjec, S. (1994). The influence of a diagnosed mental impairment on family caregiver strain. Journal of Gerontology: Social Sciences, 49, SI46-S155.

Bass, D. M., Tausig, M. B., \& Noelker, L. S. (1989). Elder impairment, social support, and caregiver strain: A framework for understanding support's effects. Journal of Applied Social Sciences, 13(1), 80-115.

16 Sebern \& Witlatch 
Beach, S. R., Schulz, R., Williamson, G. M., Miller, L. S., Weiner, M. F., \& Lance, C. E. (2005). Risk factors for potentially harmful informal caregiver behavior. Journal of the American Geriatrics Society, 53, 255-261.

Beach, S. R., Schulz, R., Yee, J. L., \& Jackson, S. (2000). Negative and positive health effects of caring for a disabled spouse: Longitudinal findings from the Caregiver Health Effects Study. Psychology and Aging, 15, 259-271.

Burg, M. M., Barefoot, J., Berkman, L., Catellier, D. J., Czajkowski, S., Saab, P., et al. (2005). Low perceived social support and post-myocardial infarction prognosis in the enhancing recovery in coronary heart disease clinical trial: The effects of treatment. Psychosomatic Medicine, 67, 879--888.

Campbell, L., \& Kashy, D. A. (2002). Estimating actor, partner, and interaction effects for dyadic data using PROC MIXED and HLM: A user-friendly guide. Personal Relationships, 9, 327-342.

Centers for Medicare \& Medicaid Services. (2007). OASIS overview. Retrieved February 3 , 2007, from http://www.cms.hhs.gov/OASIS/

Clark, P. A., Whitlatch, C. J., \& Tucke, S. S. (2005, November). Knowledge and agreement of care preferences and dyadic well-being. Paper presented at the 58th Annual Scientific Meeting of The Gerontological Society of America, Orlando, FL.

Cutrona, C., \& Cole, V. (2000). Optimizing support in the natural network. In S. Cohen, L. Underwood, \& B. Gottlieb (Eds.), Social support measurement and intervention (pp. 278308). New York: Oxford University Press.

Davey, A., \& Eggebeen, D. J. (1998). Patterns of intergenerational exchange and mental health. Journal of Gerontology: Psychological Sciences, 53B, P86-P95.

Dillon, W., \& Goldstein, M. (1984). Multivariate analysis: Methods and applications. New York: Wiley.

Eaton, W. W., VandeCreek, L., \& Jackson, T. L. (2001). Center for Epidemiologic Studies Depression Scale-Revised (CESD-R). In L. VandeCreek \& T. L. Jackson (Eds.), Innovations in clinical practice: A source book (Vol. 19, p. 295). Sarasota, FL: Professional Resource Exchange.

Feinberg, L. F., Whitlatch, C. J., \& Tucke, S. S. (2000). Making hard choices: Respecting both voices. Cleveland, $\mathrm{OH}$ : Margaret Blenkner Research Institute of Benjamin Rose.

Fornell, C., \& Larker, D. (1981). Evaluating structural equation models with unobservable variables and measurement error. Journal of Marketing Research, 18, 39-50. 
Gangler, J. E., Kane, R. L., \& Kane, R. A. (2002). Family care for older adults with disabilities. International Journal of Human Development, 54(3), 205-231.

Gayle, B. M., \& Preiss, R. W. (2002). An overview of dyadic processes in interpersonal communication. In M. Allen, R. W. Preiss, B. M. Gayle, \& N. A. Burrell (Eds.), Interpersonal communication research: Advances through meta-analysis (pp. 111-24). Mahwah, NJ: Erlbaum.

Gonzalez, R., \& Griffin, D. (1999). The correlational analysis of dyad-level data in the distinguishable case. Personal Relationships, 6, 449-469.

Kellett, U. M. (1999a). Searching for new possibilities to care: A qualitative analysis of family caring involvement in nursing homes. Nursing Inquiry, 6(1), 9-16.

Kellett, U. M. (1999b). Transition in care: Family carers' experience of nursing home placement. Journal of Advanced Nursing, 29, 1474-1481.

Kenny, D., Kashy, D., \& Cook, W. (2006). Dyadic data analysis. New York: Guilford Press.

Kline, R. (2005). Principles and practice of Structural Equation Modeling. New York: Guilford Press.

Kramer, B. J. (1997). Gain in the caregiving experience: Where are we? What next? The Gerontologist, 37, 218-232.

Krause, N. (2005). Negative interaction and heart disease in late life: Exploring variations by socioeconomic status. Journal of Aging and Health, 17, 28-55.

Lauver, D. R., Ward, S., Heidrich, S. M., Keller, M. L., Bowers, B. J., Brennan, P. F., et al. (2002). Patient-centered interventions. Research in Nursing \& Health, 25, 246-255.

Lawton, M. P., Kleban, M. H., Moss, M., Rovine, M., \& Glicksman, A. (1989). Measuring caregiving appraisal. Journal of Gerontology: Psychological Sciences, 44, P61-P71.

Lebowitz, B. D., Pearson, J. L., Schneider, L. S., Reynolds, C. F., III, Alexopoulos, G. S., Bruce, M. L., et al. (1997). Diagnosis and treatment of depression in late life. Consensus statement update. Journal of the American Medical Association, 278, 1186-1190.

Liang, J., Krause, N. M., \& Bennett, J. M. (2001). Social exchange and well-being: Is giving better than receiving? Psychology and Aging, 16, 511-523.

Martire, L. M., Lustig, A. P., Schulz, R., Miller, G. E., \& Helgeson, V. S. (2004). Is it beneficial to involve a family member? A meta-analysis of psychosocial interventions for chronic illness. Health Psychology, 23, 599-611.

Maydeu-Olivares, A. (2000). Review of MPlus. Multivariate Behavioral Research, 35, 501-505. McDowell, I. (2006). Measuring health (3rd ed.). New York: Oxford University Press. 
Menne, H. L. (2006). A stress process model of chronic illness: Understanding the well-being and decision-making involvement of individuals with dementia. Unpublished doctoral dissertation, Case Western Reserve University, Cleveland, $\mathrm{OH}$.

Muthén, L., \& Muthén, B. (2000). Software review. Structural Equation Modeling, 7, 309-317.

Muthén, L. K., \& Muthén, B. O. (Eds.). (2005). Mplus user's guide. (3rd ed.). Los Angeles: Authors.

National Institute of Nursing Research. (2004). Family caring. Retrieved September 29, 2006, from http://ninr.nih.gov/ninr/research/vol3/FamCare.html

Poulshock, S. W., \& Deimling, G. T. (1984). Families caring for elders in residence: Issues in the measurement of burden. Journal of Gerontology, 39, 230-239.

Radloff, L. S., \& Teri, L. (1986). Use of the Center for Epidemiological Studies Depression Scale with older adults. Clinical Gerontologist, 5, 119-135.

Reinhardt, J. (2001). Effects of positive and negative support received and provided on adaptation to chronic visual impairment. Applied Developmental Science, 5(2), 76-85.

Reis, H. T., \& Collins, N. (2000). Measuring relationship properties and interactions relevant to social support. In S. Cohen, L. G. Underwood, \& B. J. Gottlieb (Eds.), Social support measurement and intervention (pp. 136-192). New York: Oxford University Press.

Schafer, J. L. (1997). Analysis of incomplete multivariate data. London: Chapman \& Hall.

Sullivan, M., Levy, W. C., Russo, J. E., \& Spertus, J. A. (2004). Depression and health status in patients with advanced heart failure: A prospective study in tertiary care. Journal of Cardiac Failure, 10, 390-396.

Turvey, C. L., Schultz, K., Arndt, S., Wallace, R. B., \& Herzog, R. (2005). Prevalence and correlates of depressive symptoms in a community sample of people suffering from heart failure. Journal of the American Geriatrics Society, 50, 2003-2008.

Whitlatch, C. J., Feinberg, L., \& Tucke, S. (2005). Measuring the values and preferences for everyday care of persons with cognitive impairment and their family caregivers. The Gerontologist, 45, 370-380.

Whitlatch, C. J., Judge, K., Zarit, S. H., \& Femia, E. (in press). Dyadic counseling for family caregivers and care receivers in early state dementia. The Gerontologist.

Wills, T. (1985). Supportive functions of interpersonal relationships. In S. Cohen \& S. L. Syme (Eds.), Social support and health (pp. 61-82). New York: Academic Press.

Zarit, S., Reever, K., \& Bach-Peterson, J. (1980). Relatives of the impaired elderly: Correlates of feelings of burden. The Gerontologist, 20, 649-655.

19 Sebern \& Witlatch 


\section{Appendix}

Table 1

\section{Demographic Characteristics of 200 Matched Dyads}

\begin{tabular}{|l|c|c|}
\hline \multicolumn{1}{|c|}{ Item } & Patients & Caregivers \\
\hline Age, $M(\mathrm{SD})$ & $66(14)$ & $65(14)$ \\
\hline Gender (female), \% $(n)$ & $46(92)$ & $62(125)$ \\
\hline Race, $\%(n)$ & & \\
\hline White & $97(194)$ & $96(192)$ \\
\hline Black & $1(2)$ & $2(4)$ \\
\hline American Indian & $<1(1)$ & $<1(1)$ \\
\hline Other & $1(2)$ & $1(1)$ \\
\hline Ethnicity (Hispanic), \% (n) & & \\
\hline Education, \% (n) & $19(36)$ & $22(41)$ \\
\hline College degree & $63(119)$ & $62(117)$ \\
\hline High school diploma & $18(35)$ & $16(30)$ \\
\hline Less than high school & & \\
\hline Role relationship, \% $(n)$ & $81(162)$ & $81(162)$ \\
\hline Spouse or partner & $7(16)$ & $9(18)$ \\
\hline Son, daughter, friend & $7(14)$ & $4(8)$ \\
\hline Parent & $4(8)$ & $4(8)$ \\
\hline Other & $41(17)$ & $41(18)$ \\
\hline Years of relationship, $M($ SD) & 4 & 5 \\
\hline Limitations in activity (KPS), median & & \\
\hline Medical diagnosis, \% $(n)^{\mathrm{a}}$ & $27(53)$ & \\
\hline Circulatory & $13(25)$ & \\
\hline Musculoskeletal & $9(18)$ & \\
\hline Neoplasm & $8(16)$ & \\
\hline Disease of skin & $5(10)$ & \\
\hline Digestive system & $5(10)$ & \\
\hline Endocrine & $5(10)$ & \\
\hline Injury poisoning & $5(10)$ & \\
\hline Genitourinary system & $4(9)$ & \\
\hline Paraplegia & $3(7)$ & \\
\hline Symptoms ill defined & $3(6)$ & \\
\hline Respiratory & $3(5)$ & \\
\hline Parkinson's & $1(3)$ & \\
\hline Blood diseases & $1(2)$ & \\
\hline Congenital anomalies & & \\
\hline & & \\
\hline
\end{tabular}

Notes: KPS = Karnofsky Index of Performance Status.

${ }^{\mathrm{a}} n=184$. 
Table 2

ICC Measure of Nonindependence of Patient and Caregiver Data

\begin{tabular}{|l|c|c|c|c|c|}
\hline & & & & \multicolumn{2}{c|}{$95 \%$ Cl } \\
\hline \multicolumn{1}{|c|}{ DRS Variable } & ICC & SE & Z & Lower & Upper \\
\hline PT DRS 1 vs CG DRS 1 & $0.34^{*}$ & 0.071 & 4.81 & 0.20 & 0.48 \\
\hline PT DRS 2 vs CG DRS 2 & $0.19^{*}$ & 0.071 & 2.78 & 0.05 & 0.33 \\
\hline PT DRS 3 vs CG DRS 3 & $0.23^{*}$ & 0.071 & 3.26 & 0.09 & 0.37 \\
\hline PT DRS 4 vs CG DRS 4 & $0.14^{*}$ & 0.071 & 2.02 & 0.01 & 0.37 \\
\hline PT DRS 5 vs CG DRS 5 & $0.16^{*}$ & 0.071 & 2.30 & 0.02 & 0.30 \\
\hline PT DRS 6 vs CG DRS 6 & -0.01 & 0.071 & -0.12 & -0.14 & 0.13 \\
\hline PT DRS 7 vs CG DRS 7 & $0.24^{*}$ & 0.071 & 3.50 & 0.11 & 0.38 \\
\hline PT DRS 8 vs CG DRS 8 & $0.36^{*}$ & 0.071 & 5.12 & 0.22 & 0.50 \\
\hline PT DRS 9 vs CG DRS 9 & $0.28^{*}$ & 0.071 & 3.95 & 0.14 & 0.41 \\
\hline PT DRS 10 vs CG DRS 10 & $0.29^{*}$ & 0.071 & 4.09 & 0.15 & 0.42 \\
\hline
\end{tabular}

Notes: ICC = interclass correlation; DRS = Dyadic Relationship Scale; $\mathrm{Cl}=$ confidence interval; $\mathrm{PT}=$ patient; $\mathrm{CG}=$ caregiver.

${ }^{*} p<.05$.

Table 3

Confirmatory Factor Analysis, Patient and Caregiver Dyadic Relationship

\begin{tabular}{|c|c|c|c|c|}
\hline \multirow[b]{2}{*}{ Item } & \multicolumn{2}{|c|}{ Positive Interaction } & \multicolumn{2}{|c|}{ Dyadic Strain } \\
\hline & CG & PT & CG & PT \\
\hline I felt closer to her/him than I have in awhile. (1) & .85 & .75 & & \\
\hline I learned good things about myself. (2) & .79 & .49 & & \\
\hline I have had more patience than I had in the past. (6) & .41 & .60 & & \\
\hline I learned good things about him/her. (7) & .84 & .88 & & \\
\hline I learned nice things about other people. (9) & .47 & .64 & & \\
\hline Communication between us has improved. (10) & .76 & .84 & & \\
\hline I felt angry toward him/her. (3) & & & .87 & .84 \\
\hline $\begin{array}{l}\text { I felt depressed when I had problems with my } \\
\text { relationship. (4) }\end{array}$ & & & .86 & .58 \\
\hline I felt resentful. (5) & & & .91 & .86 \\
\hline I felt strained. (8) & & & .65 & .72 \\
\hline The patient made too many requests. ${ }^{a}(11)$ & & & .66 & \\
\hline
\end{tabular}

Notes: Extraction method was WLSMV weight least square parameter estimates. $\mathrm{CG}=$ caregiver; $\mathrm{PT}=$ patient.

${ }^{\mathrm{a}}$ On caregiver scale only. 
Table 4

Partial Standardized Regression Coefficients Between Patient DRS and Patient CES-D

\begin{tabular}{|c|c|c|c|c|}
\hline Measure & $\begin{array}{c}\text { Patient Positive } \\
\text { Dyadic Interaction }\end{array}$ & Patient Dyadic Strain & RMSEA & $\begin{array}{c}\boldsymbol{R}^{2} \text { Variance } \\
\text { Explained }\end{array}$ \\
\hline Patient CES-D & $0.20^{\star}$ & $0.52^{\star}$ & 0.06 & $23 \%$ \\
\hline
\end{tabular}

Notes: DRS = Dyadic Relationship Scale; CES-D = Center for Epidemiologic Studies-Depression scale; RMSEA = root mean square error of approximation.

${ }^{*} p<.05$.

Table 5

Partial Standardized Regression Coefficients Between Caregiver DRS and Caregiver CES-D

\begin{tabular}{|c|c|c|c|c|}
\hline Measure & $\begin{array}{c}\text { Caregiver Positive } \\
\text { Dyadic Interaction }\end{array}$ & $\begin{array}{c}\text { Caregiver } \\
\text { Dyadic Strain }\end{array}$ & RMSEA & $\begin{array}{c}\boldsymbol{R}^{2} \text { Variance } \\
\text { Explained }\end{array}$ \\
\hline Caregiver CES-D & 0.05 & $0.47^{\star}$ & 0.06 & $21 \%$ \\
\hline
\end{tabular}

Notes: $\mathrm{DRS}$ = Dyadic Relationship Scale; CES-D = Center for Epidemiologic Studies-Depression scale; RMSEA = root mean square error of approximation.

${ }^{*} p<.05$. 